\title{
Zwei Kritiken des Populismus
}

\author{
Floris Biskamp $\mathbb{D}$
}

Online publiziert: 26. Oktober 2020

(C) Der/die Autor(en) 2020

Möller, Kolja (2020): Volksaufstand \& Katzenjammer. Zur Geschichte des Populismus. Berlin: Wagenbach. 160 Seiten. $18 €$

Fassin, Eric (2019): Revolte oder Ressentiment. Über den Populismus. Köln: August. 126 Seiten. $12 €$

Mit Kolja Möllers „Volksaufstand und Katzenjammer“ sowie Eric Fassins „Revolte oder Ressentiment“" liegen zwei weitere kurze Bände von Sozialwissenschaftlern vor, die sich mit Fragen des Populismus und der politischen Strategiebildung vor allem für die politische Linke - auseinandersetzen. Beide Autoren verstehen ihre Bücher in je anderem Sinne als Kritiken des Populismus: Möller legt sein Buch als immanente Kritik an, in der er die Ansprüche, Widersprüche, Potenziale und Grenzen des Populismus auslotet; Fassin dagegen formuliert eine dezidierte Ablehnung des Populismus.

Möllers Buch ist mit knapp 120 Seiten Text relativ dünn, aber doch als historisch weit ausgreifende dialektische Auseinandersetzung mit Begriff und Gegenstand des Populismus angelegt. Der Politikwissenschaftler beginnt seine Darstellung mit dem römischen Volksaufstand aus dem Jahr 1347, in dem das grundlegende Muster von Aufstand und Populismus deutlich werde: Auf Triumph und Euphorie bei der Vertreibung der aristokratischen Eliten aus der Stadt folgten innerhalb weniger Monate erst das Abgleiten der neuen Regierung in selbstherrlichen Autoritarismus und dann das schmähliche Scheitern unter äußerem Druck - „Katzenjammer“ (S. 30) eben. Daraufhin springt der Autor ins 19. Jahrhundert, in dem die Frage des Aufstandes intensiv diskutiert wurde. Zentral für das Buch sind drei verbreitete „Fehler“ (S. 39)

F. Biskamp $(\bowtie)$

Promotionskolleg Rechtspopulistische Sozialpolitik und exkludierende Solidarität, Eberhard Karls

Universität Tübingen, Tübingen, Deutschland

E-Mail: floris.biskamp@uni-tuebingen.de 
von Volksaufständen, deren Kritik Möller aus Texten von Friedrich Engels und Karl Marx destilliert: der „,voluntaristische Fehler“ (S. 39), der „,identitäre Fehler“ (S. 44) und der ,autoritäre Fehler“ (S. 48). Voluntarismus liege vor, wenn die Bedingung für einen Erfolg des Aufstands allein in der politischen Willenskraft der Aufständischen gesehen werde, die sozialen und ökonomischen Bedingungen aber ausgeblendet blieben. Den identitären Fehler begehe, wer dem aufständischen „Volk“ eine identitäre Substanz zuschreibe - sei es in Form von Arbeitsfetisch oder in Form von Nationalismus. Zum autoritären Fehler komme es, wenn der Wille des Volkes mit der Herrschaft eines Einzelnen oder einer kleinen Gruppe identifiziert werde. Während der Voluntarismus dazu verleite, zum Scheitern verurteilte Aufstände anzuzetteln, verursachten identitärer und autoritärer Fehler eine Degeneration des Aufstands - in den Katzenjammer führten alle drei Wege.

Während der Aufstand bis ins 19. Jahrhundert hinein als politischer Umsturz gedacht worden sei, hätten sich die Bedingungen mit der Einführung der parlamentarischen Demokratie und des zunehmend allgemeinen Wahlrechts verändert. Zwar bestehe die Tendenz zur Machtmonopolisierung durch Eliten und somit auch die normative und motivationale Grundlage für den Volksaufstand fort. Dank der parlamentarischen Institutionen könne dieser jedoch eine legale institutionelle Form annehmen. Eben diese elektorale Form des Volksaufstandes heiße Populismus. Als dessen definitorisches Merkmal benennt Möller, wie heute üblich, die Mobilisierung auf Grundlage eines Gegensatzes von „Volk“ und „Elite“. Auch in der populistischparlamentarischen Form laufe der Volksaufstand weiter Gefahr, die drei genannten Fehler zu reproduzieren. Dies führe zu voluntaristischer Frustration oder zu nationalistischen und autoritären Populismen (vulgo Rechtspopulismus).

Jedoch verteidigt Möller den populistischen Anspruch, ,,das Volk“ gegen ,,die Eliten" zu mobilisieren, als genuin demokratischen Impuls und stellt sich damit gegen liberal-antipopulistische Positionen. Dabei formuliert er ein treffendes Argument gegen Jan-Werner Müller, der den populistischen Ruf „Wir sind das Volk“ ablehnt, sofern es sich um eine Entgegensetzung von „Volk“ und „Eliten“ handelt, und ihn lediglich für demokratisch hält, wenn sich darin der Ruf „Wir sind auch das Volk!“ ausdrücke, mit dem Minderheiten ihre gleichberechtigte Zugehörigkeit zum Volk einfordern. Möller betont dagegen, dass es neben derartigen Kämpfen um Inklusion auch zahlreiche Beispiele für undemokratische Elitenherrschaft gebe, gegen die der Aufstand im Namen des „Volkes“ zur Demokratisierung beitragen könne. Entsprechend steht am Ende des Buches die Vision eines ,gute[n] Aufstand[s]“ (S. 113), der aus den voluntaristischen, identitären oder autoritären Fehlern der Vergangenheit lernt, sich mit den Widersprüchen des aufständischen Handelns auseinandersetzt und dennoch (durchaus populistisch) einen elektoralen Aufstand gegen den postdemokratischen Neoliberalismus mobilisiert - ob sich der notorische Katzenjammer dabei in Gänze vermeiden lässt, bleibt offen.

Fassins Buch ist weniger breit angelegt und zielt vor allem auf die politischsoziologische Diskussion der Gegenwart. Der Soziologe entscheidet sich für keine eindeutige Definition von Populismus, was er mit der Unschärfe des Gegenstandes selbst begründet. Jedoch sieht er den Populismus ebenfalls durch eine Mobilisierung des „Volkes“ gegen „Eliten“ gekennzeichnet - und eben darin seien die Probleme angelegt. Ein einheitliches „Volk“ mit klarem „Willen“ gebe es in Wirklichkeit 
gar nicht - jedenfalls nicht als vorpolitische Größe. Wenn man dennoch versuche, dieses „Volk“ politisch zu mobilisieren, führe das nicht zu progressiver oder demokratischer Politik, sondern zur Verstärkung von nationalistischem und autoritärem Ressentiment. Progressive Politik dagegen müsse bei realen Differenzen wie denen der Klasse ansetzen, von einer Kritik sozialer Ungleichheit getrieben sein und rassistische oder sexistische Ausgrenzungen ebenso vermeiden wie autoritäre Anmaßungen. Zwar habe die Populismusbefürworterin Chantal Mouffe durchaus recht damit, dass auch progressive Politik Affekte mobilisieren müsse, es komme aber darauf an, welche Affekte. Auf der einen Seite stünde der progressiv mobilisierbare Affekt der Empörung gegenüber Ungerechtigkeit, auf der anderen Seite das nur für regressive, autoritäre Politik zu aktivierende Ressentiment gegen andere bzw. gegen unten. Weil sich die Erfolge von Trump, Le Pen und anderen Rechten vor allem aus letzterem Affekt speisten, sei jeder Versuch, ihre WählerInnen durch einen linken Populismus abzuwerben, zum Scheitern verurteilt.

In der Frage, welche Strategie normativ wünschenswert ist, stehen sich die beiden Autoren relativ nahe: Beide befürworten eine Politisierung sozioökonomischer Fragen von links sowie eine progressive Positionierung in soziokulturellen Fragen; Möller unter der Bedingung, dass diese Mobilisierung die voluntaristischen, identitären und autoritären Fehler vermeidet, Fassin unter der Bedingung, dass sie keine Ressentiments mobilisiert und nicht versucht ,das Volk“ anzusprechen. Auf die Politik des linken Flügels der Demokratischen Partei in den Vereinigten Staaten könnten sich die beiden wohl einigen - mit dem nominellen Unterschied, dass Fassin dabei nicht von Populismus sprechen würde.

Einigkeit besteht auch in der Ablehnung populistischer Projekte, die ein linkes Selbstverständnis mit einem Bekenntnis zur Nation und ihren Grenzen verbinden. Bei Möller gilt dies als ein Beispiel für den identitären Fehler, bei Fassin als Mobilisierung eines nationalistischen, rassistischen und genuin rechten Ressentiments. Jedoch bietet keiner von beiden ein überzeugendes Argument gegen die im ,nationalen“ Linkspopulismus gängige und zum Beispiel von Andreas Nölke vertretene These, der zufolge es für die populären Klassen schlichtweg rational sei, sich nationale Grenzen zu wünschen, weil sozialstaatliche Umverteilung in einer Welt ohne Grenzen unmöglich würde. Demnach wäre ein populistischer Protest gegen neoliberale Elitenherrschaft, der nicht auch die Grenzen der Nation guthieße, gar nicht zielführend. Diese These müsste man entweder empirisch widerlegen oder man müsste anerkennen, dass daraus ein Dilemma folgt, zu dem man sich verhalten muss. Denn auch wenn man diese These akzeptiert, kann man mit Möller und Fassin daran festhalten, dass eine solche Mobilisierung in der Praxis eine Mobilisierung nationalistischer Ressentiments sowie normativ nichtrechtfertigbare Ausschlüsse Dritter hervorbringt.

Fassins Buch leidet etwas unter dem Alter des französischen Originaltexts - bis auf das 28-seitige Nachwort stammt der nun in Übersetzung vorliegende Text aus dem Jahr 2017. Das führt dazu, dass man die meisten Argumente so oder so ähnlich schon von anderen gelesen hat. Zudem wirkt die Auseinandersetzung an einigen Stellen etwas oberflächlich. Beispielsweise scheint die säuberliche Unterscheidung in progressive und regressive Affekte, der dann noch eine Unterscheidung von WählerInnengruppen entsprechen soll, wenig überzeugend - als ob der Affekthaushalt 
von Menschen nicht von Grund auf ambivalent wäre. Sucht man jedoch nach einer pointierten Zusammenfassung einer sozialwissenschaftlich informierten Argumentation gegen populistische Politik als linke Strategie, wird man im Buch fündig.

Möller formuliert zwar ebenfalls keine Thesen, die das intensiv beackerte Feld des Populismus revolutionieren würden, in der Darstellungsweise ist sein Band dennoch innovativ, pointiert und somit ausgesprochen lesenswert. Ob es sich wirklich, wie vom Autor beansprucht, um eine immanente Kritik des Populismus handelt, kann man in Zweifel ziehen. Das am Ende präsentierte Verständnis des ,guten Aufstands" lässt erahnen, dass ihm ein normatives Verständnis von demokratischer Politik zugrunde liegt, an dem der Populismus eher gemessen wird, als dass es aus seiner immanenten Kritik heraus entwickelt worden wäre. Mit einem stärker republikanisch-kommunitaristisch geprägten normativen Verständnis von Demokratie wäre man womöglich zu anderen Ergebnissen gekommen. Zudem wäre es wünschenswert, etwas mehr über das Verhältnis zu lesen, in dem der „gute Aufstand“ $\mathrm{zu}$ liberal-rechtsstaatlichen Institutionen steht.

Funding Open Access funding enabled and organized by Projekt DEAL.

Open Access Dieser Artikel wird unter der Creative Commons Namensnennung 4.0 International Lizenz veröffentlicht, welche die Nutzung, Vervielfältigung, Bearbeitung, Verbreitung und Wiedergabe in jeglichem Medium und Format erlaubt, sofern Sie den/die ursprünglichen Autor(en) und die Quelle ordnungsgemäß nennen, einen Link zur Creative Commons Lizenz beifügen und angeben, ob Änderungen vorgenommen wurden.

Die in diesem Artikel enthaltenen Bilder und sonstiges Drittmaterial unterliegen ebenfalls der genannten Creative Commons Lizenz, sofern sich aus der Abbildungslegende nichts anderes ergibt. Sofern das betreffende Material nicht unter der genannten Creative Commons Lizenz steht und die betreffende Handlung nicht nach gesetzlichen Vorschriften erlaubt ist, ist für die oben aufgeführten Weiterverwendungen des Materials die Einwilligung des jeweiligen Rechteinhabers einzuholen.

Weitere Details zur Lizenz entnehmen Sie bitte der Lizenzinformation auf http://creativecommons.org/ licenses/by/4.0/deed.de. 